\title{
Mathematical analysis of strain localization
}

\author{
Milan Jirásek \\ Department of Mechanics \\ Faculty of Civil Engineering \\ Czech Technical University in Prague \\ 16629 Prague, Czech Republic \\ Milan.Jirasek@epfl.ch
}

\begin{abstract}
First it is shown by a simple one-dimensional example that stress-strain laws with softening cannot provide an objective description of response on the structural level. The phenomenon of discontinuous bifurcation from a uniform state is then analyzed in a general threedimensional setting. Localization conditions for isotropic damage models are derived in the general form and then specialized for models with damage driven by equivalent strain dependent on the stored elastic energy, on the maximum principal effective stress, or on the positive part of the strain tensor.

RÉSUMÉ. D'abord on montre à l'aide d'un exemple unidimensionnel que les lois constitutives avec adoucissement ne peuvent pas décrire la réponse au niveau de la structure dans une manière objective. Ensuite, le phénomène de la bifurcation discontinue à partir d'un état uniforme est analysé dans le context général tridimensionnel. Les conditions de localisation pour les modèles d'endommagement isotrope sont déduites dans leur forme générale, qui est ensuite spécialisée pour les modèles avec endommagement dépendant de l'énergie élastique, de la valeur principale maximale de la contrainte effective, ou de la partie positive du tenseur de déformation.
\end{abstract}

KEYWORDS: strain localization, softening, discontinuous bifurcation.

MOTS-CLÉS : localisation de la déformation, adoucissement, bifurcation discontinue.

REGC - 11/2007. Damage and fracture in geomaterials, pages 977 à 991 


\section{Inobjectivity of standard strain-softening continuum}

The idea of modeling cracking concrete and other quasibrittle materials as strainsoftening continua, which emerged in the seventies, was not immediately accepted by the entire scientific community. It was necessary to overcome a number of objections regarding the ill-posedness and lack of objectivity of such formulations. Indeed, most of the early analyses were not truly objective and, upon mesh refinement, their results would not have converged to a meaningful solution. Instead of going into theoretical details, let us explain the nature of the problem by a simple example.

Consider a straight bar of a constant cross section $A$ and of total length $L$ under uniaxial tension; see Figure 1a. The material is assumed to obey a simple stress-strain law with linear elasticity up to the peak stress, $f_{t}$, followed by linear softening (Figure $2 \mathrm{a}$ ). The strain at which the transmitted stress completely disappears is denoted by $\varepsilon_{f}$. The peak stress is developed at strain $\varepsilon_{0}=f_{t} / E$ where $E$ is Young's modulus.

If the bar is loaded in tension by an applied displacement $u$ of the right support, the response remains linear elastic up to $u_{0}=L \varepsilon_{0}$. At this state, the force transmitted by the bar (reaction at the support) attains its maximum value, $F_{0}=A f_{t}$. After that, the resistance of the bar starts decreasing. At each cross section, stress can decrease either at increasing strain (softening) or at decreasing strain (elastic unloading). The static equilibrium equation implies that the stress profile must remain uniform along the bar. However, at any given stress level $\bar{\sigma}$ between zero and $f_{t}$, there exist two values of strain for which the constitutive equation is satisfied (Figure 2a), and so the strain profile does not have to remain uniform. In fact, any piecewise constant strain distribution that jumps between the two strain values $\varepsilon_{s}$ and $\varepsilon_{u}$ (respectively corresponding to softening and unloading) represents a valid solution; see Figure $1 \mathrm{~b}$. Let us denote by $L_{s}$ the cumulative length of the softening regions and by $L_{u}=L-L_{s}$ the cumulative length of the unloading regions. When stress decreases to zero, the strain in the softening region is $\varepsilon_{s}=\varepsilon_{f}$ and the strain in the unloading region is $\varepsilon_{u}=0$; the total elongation of the bar is therefore $u_{f}=L_{s} \varepsilon_{s}+L_{u} \varepsilon_{u}=L_{s} \varepsilon_{f}$.

(a)

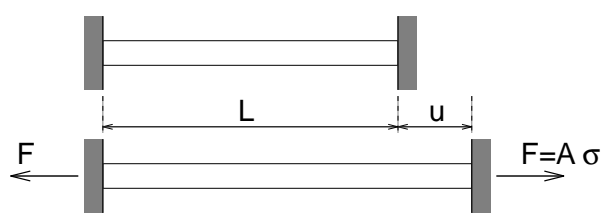

(b)

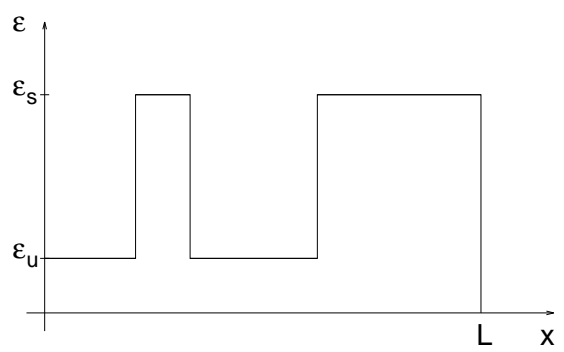

Figure 1. (a) Bar under uniaxial tension, (b) piecewise constant strain profile 
(a)

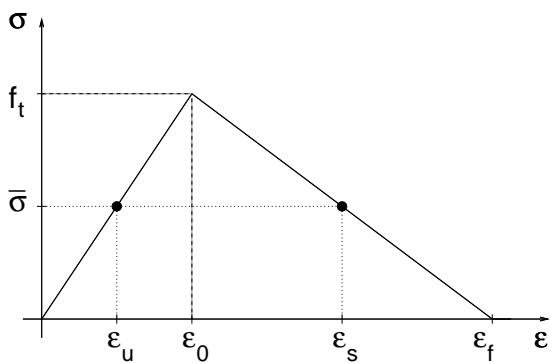

(b)

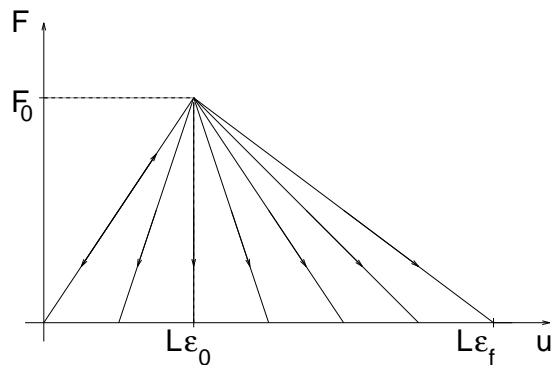

Figure 2. (a) Stress-strain diagram with linear softening, (b) fan of possible post-peak branches of the load-displacement diagram

Now the tricky point is that the length $L_{s}$ is undetermined, and it can be anything between zero and $L$. This means that the problem has infinitely many solutions, and the corresponding post-peak branches of the load-displacement diagram fill the fan shown in Figure $2 \mathrm{~b}$. This fan is bounded on one side by the solution with uniform softening $\left(L_{s}=L, u_{f}=L \varepsilon_{f}\right)$ and on the other side by the solution with uniform unloading $\left(L_{u}=L, u_{f}=0\right)$. The latter limit correctly represents the case when the bar is unloaded just before any softening occurs. All the other solutions describe a possible process in which a part of the bar is damaged and the bar loses its structural integrity. It is not clear which of these solutions is "correct" in the sense that it reflects the actual failure process.

The ambiguity is removed if imperfections are taken into account. Real material properties and sectional dimensions cannot be perfectly uniform. Suppose that the strength in a small region is slightly lower than in the remaining portion of the bar. When the applied stress reaches the reduced strength, softening starts and the stress decreases. Consequently, the material outside the weaker region must unload elastically, because its strength has not been exhausted. This leads to the conclusion that the size of the softening region is dictated by the size of the region with minimum strength. Such a region can be arbitrarily small, and so the corresponding softening branch is arbitrarily close to the elastic branch of the load-displacement diagram. Thus the standard strain-softening continuum formulation leads to a solution that has several pathological features:

1) The softening region is infinitely small.

2) The load-displacement diagram always exhibits snapback, independently of the size of the structure and of the ductility of the material.

3) The total amount of energy dissipated during the failure process is zero.

From the mathematical point of view, these annoying features are related to the loss of ellipticity of the governing differential equation. The boundary value problem be- 
comes ill-posed, i.e., it does not have a unique solution with continuous dependence on the given data. From the numerical point of view, ill-posedness is manifested by pathological sensitivity of the results to the size of finite elements. This will be illustrated by an example in Jirásek (2007), and various remedies that lead to objective, mesh-insensitive results will be described. In the present paper we focus on a deeper analysis of conditions under which we can expect such pathological behavior of classical continuum models.

\section{Discontinuous bifurcations-general theory}

The simple one-dimensional example presented in the previous section illustrated the essence of the problem with localization of inelastic strain into a process zone of an arbitrarily small width. In one dimension, localization occurs when the peak of the stress-strain diagram is reached, independently of the specific constitutive model used. Formulations based on damage mechanics, smeared cracks or softening plasticity all lead to the same type of behavior as soon as the tangent material stiffness ceases to be positive. In multiple dimensions, the analysis of the localization process is more complicated and the derivation of criteria for potential onset of localization represents a challenging mathematical problem.

The fundamental question is under which conditions the inelastic strain increments can localize in one or more narrow bands separated from the remaining part of the body by weak discontinuity surfaces. Across such surfaces, the displacement field remains continuous but the strain field can have a jump. At the onset of localization, the current strains are still continuous and the jump appears only in the strain rates. Let us determine the necessary conditions for the existence of such a solution, using the classical localization analysis inspired by the early works of Hadamard (1903) and Hill (1958) and developed, among others, for plasticity by Rudnicky et al. (1975) and Ottosen et al. (1991) and for damage by Rizzi et al. (1996).

Our analysis will be restricted to one point $\boldsymbol{x}_{d}$ of the discontinuity surface $S_{d}$ at incipient loss of strain continuity. The discontinuity surface splits the body (at least locally) into subdomains $V^{+}$and $V^{-}$and its direction is characterized by the unit vector $\boldsymbol{n}$, oriented towards $V^{+}$; see Figure 3. If point $\boldsymbol{x}_{d}$ is approached from $V^{+}$or from $V^{-}$, those fields that are discontinuous across $S_{d}$ tend to different limits, further denoted by superscripts ${ }^{+}$and ${ }^{-}$, resp. So for instance $\dot{\boldsymbol{\sigma}}^{+}$and $\dot{\boldsymbol{\sigma}}^{-}$respectively denote the stress rates on the "positive" and "negative" side of the discontinuity surface just next to point $\boldsymbol{x}_{d}$, and their difference $[[\dot{\boldsymbol{\sigma}}]]=\dot{\boldsymbol{\sigma}}^{+}-\dot{\boldsymbol{\sigma}}^{-}$is the jump of the strain rate.

Even though the stress and strain rates can be discontinuous across $S_{d}$, the corresponding jumps are not completely arbitrary. The stress rate jump $[[\dot{\boldsymbol{\sigma}}]]$ is constrained by the traction continuity condition and the strain rate jump $[[\dot{\varepsilon}]]$ by the displacement continuity condition. If the tensors are decomposed into their in-plane and out-ofplane parts (with respect to the plane tangent to the discontinuity surface), the traction continuity condition means that the out-of-plane stress rates must be continuous and 


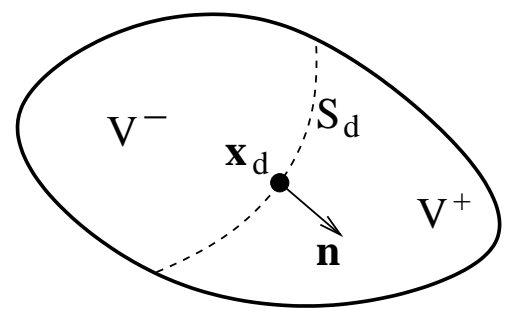

Figure 3. Body split by a potential discontinuity surface

the displacement continuity condition implies that the in-plane strain rates must be continuous. The jumps can appear only in the in-plane stress rates and out-of-plane strain rates.

The traction continuity condition is easily written as

$$
\boldsymbol{n} \cdot \dot{\boldsymbol{\sigma}}^{+}=\boldsymbol{n} \cdot \dot{\boldsymbol{\sigma}}^{-}
$$

Formal mathematical representation of the displacement continuity condition in terms of strain rates is less obvious. We can start from the observation that the spatial gradient of a given displacement component, e.g. of $u_{1}$, must have continuous projections onto the tangent plane (to the discontinuity surface) and only the normal component of this first-order tensor can have a jump. So we can write

$$
\left(\frac{\partial \dot{u}_{1}}{\partial \boldsymbol{x}}\right)^{+}=\left(\frac{\partial \dot{u}_{1}}{\partial \boldsymbol{x}}\right)^{-}+c \boldsymbol{n}
$$

where $c$ is an arbitrary multiplier. Extending this relation to the entire displacement gradient, we obtain

$$
\left(\frac{\partial \dot{\boldsymbol{u}}}{\partial \boldsymbol{x}}\right)^{+}=\left(\frac{\partial \dot{\boldsymbol{u}}}{\partial \boldsymbol{x}}\right)^{-}+\boldsymbol{c} \otimes \boldsymbol{n}
$$

where $c$ is an arbitrary first-order tensor. To give to $c$ a more specific physical meaning, we present it in the form $\boldsymbol{c}=\dot{e} \boldsymbol{m}$ where $\dot{e}=\|\boldsymbol{c}\|$ is the magnitude of the jump term and $\boldsymbol{m}=\boldsymbol{c} /\|\boldsymbol{c}\|$ is a unit first-order tensor called the polarization vector. The angle between unit vectors $\boldsymbol{m}$ and $\boldsymbol{n}$ characterizes the failure mode, ranging from tensile splitting (mode I) with $\boldsymbol{m}=\boldsymbol{n}$ to shear slip (mode II) with $\boldsymbol{m}$ perpendicular to $n$; see Figure 4 b,c. In the small-strain theory, with strains defined as the symmetric part of the displacement gradient, relation [3] is easily rewritten in terms of the strain rates:

$$
\dot{\boldsymbol{\varepsilon}}^{+}=\dot{\boldsymbol{\varepsilon}}^{-}+\frac{1}{2}(\boldsymbol{m} \otimes \boldsymbol{n}+\boldsymbol{n} \otimes \boldsymbol{m}) \dot{e}
$$

The stress and strain rates should now be linked by the constitutive law. The rate form of the stress-strain law is normally written as $\dot{\sigma}=\boldsymbol{D}: \dot{\varepsilon}$ where $\boldsymbol{D}$ is the tangent 
stiffness tensor. The tangent stiffness depends on the current state of the material, which is initially the same on both sides of the incipient discontinuity, but it may also depend on the strain rate. For rate-independent models, dependence on the magnitude of the strain rate is excluded, but the tangent stiffness may depend on the oriented direction of the strain rate. For instance, for damage models certain strain rate directions lead to damage growth and others to elastic unloading at constant damage, and these two cases are characterized by two different tangent stiffness tensors. Since the strain rates on both sides of the discontinuity are different, we admit that in general the tangent stiffness tensors can be different as well, and so the stress-strain equations read

$$
\dot{\sigma}^{+}=D^{+}: \dot{\varepsilon}^{+}, \quad \dot{\sigma}^{-}=D^{-}: \dot{\varepsilon}^{-}
$$

Substituting this into the traction continuity condition [1] and exploiting relation [4] and the minor symmetry of the stiffness tensor, we obtain

$$
\boldsymbol{n} \cdot \boldsymbol{D}^{+}: \dot{\varepsilon}^{-}+\boldsymbol{n} \cdot \boldsymbol{D}^{+}:(\boldsymbol{m} \otimes \boldsymbol{n}) \dot{e}=\boldsymbol{n} \cdot \boldsymbol{D}^{-}: \dot{\varepsilon}^{-}
$$

Finally, moving the first term to the right-hand side leads to

$$
\left(\boldsymbol{n} \cdot \boldsymbol{D}^{+} \cdot \boldsymbol{n}\right) \cdot \boldsymbol{m} \dot{e}=\boldsymbol{n} \cdot\left(\boldsymbol{D}^{-}-\boldsymbol{D}^{+}\right): \dot{\varepsilon}^{-}
$$

This is the most general equation describing an incipient weak discontinuity. Considerable simplification is achieved if the tangent stiffness tensors on both sides of the discontinuity are the same, $D^{+}=D^{-}=D$. In this case, the right-hand side of [7] vanishes and, since a true discontinuity is obtained only with $\dot{e} \neq 0$, the equation reduces to

$$
(\boldsymbol{n} \cdot \boldsymbol{D} \cdot \boldsymbol{n}) \cdot \boldsymbol{m}=\mathbf{0}
$$

The second-order tensor

$$
Q=n \cdot D \cdot n
$$

is called the localization tensor. ${ }^{1}$ From [8] it is clear that, at incipient formation of a weak discontinuity, the localization tensor is singular and the polarization vector $\boldsymbol{m}$ is its eigenvector associated with eigenvalue zero. This brings us to the classical localization condition

$$
\operatorname{det} \boldsymbol{Q}=0
$$

From the mathematical point of view, singularity of the localization tensor indicates the so-called loss of ellipticity.

1. The localization tensor $Q$ is sometimes referred to as the acoustic tensor, because if the tangent stiffness tensor $\boldsymbol{D}$ is taken as the elastic stiffness tensor $\boldsymbol{D}_{\mathrm{e}}$, the eigenvalues of the corresponding acoustic tensor $\boldsymbol{Q}_{\mathrm{e}}=\boldsymbol{n} \cdot \boldsymbol{D}_{\mathrm{e}} \cdot \boldsymbol{n}$ divided by the mass density are squares of the speeds of elastic waves propagating in the direction of $\boldsymbol{n}$. The corresponding eigenvalues are polarization vectors that determine the type of waves (longitudinal, transversal, mixed). 
(a)

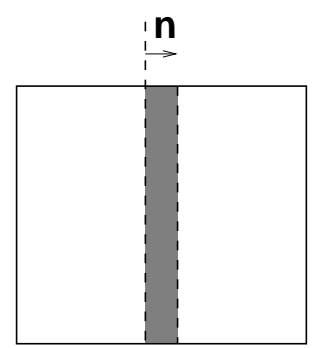

(b)

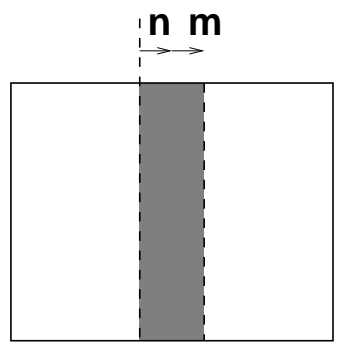

(c)

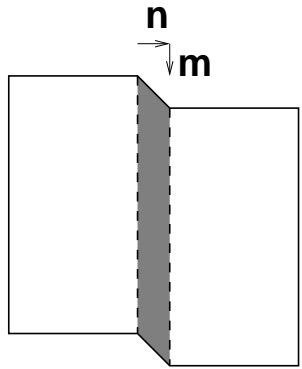

Figure 4. a) Body with a localization band, b) tensile splitting (mode I), c) shear slip (mode II)

The localization tensor defined in [9] depends on the tangent stiffness tensor $\boldsymbol{D}$ and on the unit normal to the discontinuity surface, $\boldsymbol{n}$. With certain exceptions (e.g. models with multiple loading conditions or incrementally nonlinear models), the tangent stiffness can be considered as dependent on the current state only and thus known. The vector $\boldsymbol{n}$, however, is not given in advance. Therefore, localization analysis consists in searching for a unit vector $\boldsymbol{n}$ for which the localization tensor becomes singular. If such a vector does not exist, the strain field must remain continuous. Singularity of the localization tensor for a certain vector $\boldsymbol{n}$ indicates that a strain jump can develop across a surface with normal $\boldsymbol{n}$. One should bear in mind that this condition for the appearance of a weak discontinuity is only necessary but not sufficient because the localization analysis presented here is purely local, restricted to the level of a material point and its infinitely small neighborhood. Whether the discontinuity surface indeed develops in a finite body depends on the state of the surrounding material and on the boundary conditions. Nevertheless, analysis of the localization tensor is widely used as a powerful indicator of potential discontinuous failure modes.

In the artificial but illustrative case of a body under uniform stress, the localization condition $\operatorname{det} \boldsymbol{Q}=0$ can be satisfied at all points simultaneously. The local discontinuities potentially appearing at individual points can then easily merge into global discontinuity planes. For instance, one can imagine solutions with a band (layer) under uniform strain rate, enclosed by two parallel discontinuity planes that separate the band from the remaining part of the body, in which the strain rate is also uniform but different from the strain rate inside the band; see Figure 4a. If the polarization vector $\boldsymbol{m}$ is aligned with the normal vector $\boldsymbol{n}$, the difference between the strain rates inside and outside the band corresponds to stretching of the band in the normal direction; see Figure $4 \mathrm{~b}$. This discontinuous mode is a precursor to splitting failure and is denoted as mode I. On the other hand, if the polarization vector $\boldsymbol{m}$ is perpendicular to vector $\boldsymbol{n}$, i.e., parallel with the discontinuity planes, the failure occurs by shear slip and this 
is referred to as mode II; see Figure 4c. For general vectors $\boldsymbol{m}$, failure is of a mixed type, and the angle between $\boldsymbol{m}$ and $\boldsymbol{n}$ indicates whether the failure mode is closer to tensile splitting or to shear slip.

\section{Localization analysis of damage models}

\subsection{Localization condition for isotropic damage models}

As a characteristic example, we will consider a simple isotropic damage model with one scalar damage variable $\omega$, described by the stress-strain law

$$
\boldsymbol{\sigma}=(1-\omega) \boldsymbol{D}_{e}: \boldsymbol{\varepsilon}
$$

damage law

$$
\omega=g(\kappa)
$$

and loading-unloading conditions

$$
f(\varepsilon, \kappa) \equiv \varepsilon_{\text {eq }}(\varepsilon)-\kappa \leq 0, \quad \dot{\kappa} \geq 0, \quad f(\varepsilon, \kappa) \dot{\kappa}=0
$$

in which $f$ is the damage loading function, $g$ is the damage evolution function, $\varepsilon_{\text {eq }}$ is a scalar measure of the strain level called the equivalent strain, and $\kappa$ is an internal variable that corresponds to the maximum level of equivalent strain ever reached in the previous history of the material. The choice of the specific expression for the equivalent strain directly affects the shape of the elastic domain in the strain space and, as will be shown in Section 3.3, also the localization properties of the model.

From the rate form of the basic equations it is easy to derive the (elastic-damaged) tangent stiffness tensor

$$
\boldsymbol{D}_{e d}=(1-\omega) \boldsymbol{D}_{\mathrm{e}}-g^{\prime} \overline{\boldsymbol{\sigma}} \otimes \boldsymbol{\eta}
$$

Here, $\boldsymbol{D}_{\mathrm{u}}=(1-\omega) \boldsymbol{D}_{\mathrm{e}}$ is the unloading stiffness, $g^{\prime}=\mathrm{d} g / \mathrm{d} \kappa$ is the derivative of the damage function $g, \overline{\boldsymbol{\sigma}}=\boldsymbol{D}_{e}: \boldsymbol{\varepsilon}$ is the effective stress, and $\boldsymbol{\eta}=\partial \varepsilon_{\mathrm{eq}} / \partial \varepsilon$ is a second order tensor obtained by differentiation of the expression for equivalent strain with respect to the strain tensor.

Specific conditions for discontinuous bifurcations depend on the particular choice of the equivalent strain definition, but their general form can be elaborated for the entire class of one-parameter isotropic damage models. Recall that incipient localization is characterized by the unit normal vector $\boldsymbol{n}$ defining the discontinuity plane, by the unit polarization vector $\boldsymbol{m}$ characterizing the discontinuity mode, and by rates $\dot{e}$ and $\dot{\varepsilon}^{-}$. The solution must satisfy condition [7], in which $\boldsymbol{D}^{+}$and $\boldsymbol{D}^{-}$are the tangent stiffness tensors corresponding to the processes taking place at the positive and negative sides of the discontinuity plane. Only if these tensors coincide, the localization condition can be simplified to [8] or [10]. For a damage model with a single loading 
function, the tangent stiffness at a given material state can be either $\boldsymbol{D}_{\mathrm{u}}=(1-\omega) \boldsymbol{D}_{\mathrm{e}}$ if the strain increment leads to elastic unloading, or $\boldsymbol{D}_{e d}$ if the strain increment leads to damage growth. In total we should consider $2 \times 2=4$ combinations of stiffnesses $D^{+}$and $D^{-}$, but since we are free to chose which side is considered as positive, it is sufficient to analyze the following three cases:

Case 1: Unloading on both sides. In this case we set $\boldsymbol{D}^{+}=\boldsymbol{D}^{-}=\boldsymbol{D}_{\mathrm{u}}$ and localization can occur only if the localization tensor

$$
\boldsymbol{Q}_{\mathrm{u}}=\boldsymbol{n} \cdot \boldsymbol{D}_{\mathrm{u}} \cdot \boldsymbol{n}=(1-\omega) \boldsymbol{n} \cdot \boldsymbol{D}_{\mathrm{e}} \cdot \boldsymbol{n}=(1-\omega) \boldsymbol{Q}_{\mathrm{e}}
$$

is singular. However, it is easy to show that the (elastic) acoustic tensor

$$
\boldsymbol{Q}_{\mathrm{e}}=\boldsymbol{n} \cdot \boldsymbol{D}_{\mathrm{e}} \cdot \boldsymbol{n}=\boldsymbol{n} \cdot\left(\lambda \boldsymbol{\delta} \otimes \boldsymbol{\delta}+2 \mu \boldsymbol{I}_{s}\right) \cdot \boldsymbol{n}=(\lambda+\mu) \boldsymbol{n} \otimes \boldsymbol{n}+\mu \boldsymbol{\delta}
$$

is positive definite (for all unit vectors $\boldsymbol{n}$ ), and since $\boldsymbol{Q}_{\mathrm{u}}$ is its positive multiple, the localization condition $\operatorname{det} \boldsymbol{Q}_{\mathrm{u}}=0$ cannot be satisfied. This confirms our intuitive expectation that discontinuous bifurcation must be related to damage growth.

Case 2: Damage growth on both sides. The analysis is again based on the determinant of the localization tensor, but this time we set $\boldsymbol{D}^{+}=\boldsymbol{D}^{-}=\boldsymbol{D}_{\text {ed }}$ and evaluate

$$
\boldsymbol{Q}_{\mathrm{ed}}=(1-\omega) \boldsymbol{Q}_{\mathrm{e}}-g^{\prime}(\boldsymbol{n} \cdot \overline{\boldsymbol{\sigma}}) \otimes(\boldsymbol{\eta} \cdot \boldsymbol{n})=\boldsymbol{Q}_{\mathrm{u}}-g^{\prime} \overline{\boldsymbol{\sigma}}_{\mathrm{n}} \otimes \boldsymbol{\eta}_{\mathrm{n}}
$$

where we have denoted for simplicity $\overline{\boldsymbol{\sigma}}_{\mathrm{n}}=\boldsymbol{n} \cdot \overline{\boldsymbol{\sigma}}$ and $\boldsymbol{\eta}_{\mathrm{n}}=\boldsymbol{\eta} \cdot \boldsymbol{n}$. In fact, $\overline{\boldsymbol{\sigma}}_{\mathrm{n}}$ has a direct physical meaning - it is the effective traction vector on the potential discontinuity plane.

Singularity of $\boldsymbol{Q}_{\text {ed }}$ occurs if there exists a nonzero vector $\boldsymbol{m}$ such that $\boldsymbol{Q}_{\text {ed }} \cdot \boldsymbol{m}=\mathbf{0}$, which is equivalent to

$$
\boldsymbol{Q}_{\mathrm{u}} \cdot \boldsymbol{m}=g^{\prime} \overline{\boldsymbol{\sigma}}_{\mathrm{n}}\left(\boldsymbol{\eta}_{\mathrm{n}} \cdot \boldsymbol{m}\right)
$$

As shown before, $\boldsymbol{Q}_{\mathrm{u}}$ is always positive definite and therefore invertible (as long as $\omega<1$ ). The right-hand side of [18] is a scalar multiple of vector $\overline{\boldsymbol{\sigma}}_{\mathrm{n}}$, and so if a vector $\boldsymbol{m}$ satisfying [18] exists at all, it must be a scalar multiple of $\boldsymbol{Q}_{\mathrm{u}}^{-1} \cdot \overline{\boldsymbol{\sigma}}_{\mathrm{n}}$. Therefore, let us set $\boldsymbol{m}=a \boldsymbol{Q}_{\mathrm{u}}^{-1} \cdot \overline{\boldsymbol{\sigma}}_{\mathrm{n}}$ where $a$ is a scalar multiplier to be determined, and let us verify by substituting back into [18] whether this equation can be satisfied. The resulting condition is easily transformed into

$$
\left(1-g^{\prime} \boldsymbol{\eta}_{\mathrm{n}} \cdot \boldsymbol{Q}_{\mathrm{u}}^{-1} \cdot \overline{\boldsymbol{\sigma}}_{\mathrm{n}}\right) a \overline{\boldsymbol{\sigma}}_{\mathrm{n}}=\mathbf{0}
$$

For $a=0$ or $\overline{\boldsymbol{\sigma}}_{\mathrm{n}}=\mathbf{0}$ we would get the trivial solution $\boldsymbol{m}=\mathbf{0}$, which does not correspond to a real bifurcation. A nontrivial solution exists only if the expression in parentheses on the left-hand side of [19] vanishes, i.e., if

$$
g^{\prime} \boldsymbol{\eta}_{\mathrm{n}} \cdot \boldsymbol{Q}_{\mathrm{u}}^{-1} \cdot \overline{\boldsymbol{\sigma}}_{\mathrm{n}}=1
$$


The product $\boldsymbol{\eta}_{\mathrm{n}} \cdot \boldsymbol{Q}_{\mathrm{u}}^{-1} \cdot \overline{\boldsymbol{\sigma}}_{\mathrm{n}}$ depends on the elastic constants, on the current state of the material and on the assumed direction of discontinuity plane. For a given material state, it is continuous as a function of the unit vector $\boldsymbol{n}$ and has a maximum with respect to $\boldsymbol{n}$. Variable $g^{\prime}$ (the derivative of the damage function with respect to the equivalent strain) indicates how "dramatically" damage evolves. If $g^{\prime}$ is sufficiently small, condition [20] is not satisfied for any $\boldsymbol{n}$ and discontinuous bifurcations are excluded. The minimum value of $g^{\prime}$ that is needed to allow a discontinuous bifurcation is

$$
g_{\text {crit }}^{\prime}=\frac{1}{\max _{\|\boldsymbol{n}\|=1}\left(\boldsymbol{\eta}_{\mathrm{n}} \cdot \boldsymbol{Q}_{\mathrm{u}}^{-1} \cdot \overline{\boldsymbol{\sigma}}_{\mathrm{n}}\right)}
$$

If $g^{\prime}<g_{c r i t}^{\prime}$, the localization tensor $\boldsymbol{Q}_{\text {ed }}$ is regular for all possible directions $\boldsymbol{n}$, and a discontinuous bifurcation is excluded. If $g^{\prime}=g_{\text {crit }}^{\prime}$, the localization tensor $\boldsymbol{Q}_{\text {ed }}$ is singular for that particular direction $\boldsymbol{n}$ which maximizes $\boldsymbol{\eta}_{\mathrm{n}} \cdot \boldsymbol{Q}_{\mathrm{u}}^{-1} \cdot \overline{\boldsymbol{\sigma}}_{\mathrm{n}}$, and a discontinuity across a plane perpendicular to that direction can start evolving. Finally, if $g^{\prime}>g_{\text {crit }}^{\prime}$, there exist infinitely many directions $\boldsymbol{n}$ for which $\boldsymbol{Q}_{\mathrm{ed}}$ is singular, and the discontinuity can evolve even "more easily".

Case 3: Damage growth on one side only. This is the most complicated case, because the stiffness tensors on both sides are different and we need to start from condition [7] instead of simply checking the determinant of the localization tensor. Nevertheless, the analysis can follow the same idea as in the previous case, it is only more tedious. We skip the algebraic details and proceed to the resulting condition that must be satisfied by the rates $\dot{\varepsilon}^{-}$and $\dot{e}$, respectively characterizing the strain rate on the unloading side and the magnitude of the strain jump:

$$
g^{\prime}\left(\boldsymbol{\eta}_{\mathrm{n}} \cdot \boldsymbol{Q}_{\mathrm{u}}^{-1} \cdot \overline{\boldsymbol{\sigma}}_{\mathrm{n}}+\left\|\boldsymbol{Q}_{\mathrm{u}}^{-1} \cdot \overline{\boldsymbol{\sigma}}_{\mathrm{n}}\right\| \frac{\boldsymbol{\eta}: \dot{\boldsymbol{\varepsilon}}^{-}}{\dot{e}}\right)=1
$$

The solution is admissible only if the equivalent strain is nondecreasing on the unloading side and increasing on the other side, which can be written as

$$
\boldsymbol{\eta}: \dot{\boldsymbol{\varepsilon}}^{-} \leq 0, \quad \boldsymbol{\eta}: \dot{\boldsymbol{\varepsilon}}^{-}+\dot{e} \boldsymbol{m} \cdot \boldsymbol{\eta}_{\mathrm{n}}>0
$$

where the polarization vector is given by

$$
\boldsymbol{m}=\frac{\boldsymbol{Q}_{\mathrm{u}}^{-1} \cdot \overline{\boldsymbol{\sigma}}_{\mathrm{n}}}{\left\|\boldsymbol{Q}_{\mathrm{u}}^{-1} \cdot \overline{\boldsymbol{\sigma}}_{\mathrm{n}}\right\|}
$$

From condition [22] we can express

$$
\begin{aligned}
g^{\prime} & =\frac{\dot{e}}{\boldsymbol{\eta}_{\mathrm{n}} \cdot \boldsymbol{Q}_{\mathrm{u}}^{-1} \cdot \overline{\boldsymbol{\sigma}}_{\mathrm{n}} \dot{e}+\left\|\boldsymbol{Q}_{\mathrm{u}}^{-1} \cdot \overline{\boldsymbol{\sigma}}_{\mathrm{n}}\right\| \boldsymbol{\eta}: \dot{\boldsymbol{\varepsilon}}^{-}}= \\
& =\frac{1}{\boldsymbol{\eta}_{\mathrm{n}} \cdot \boldsymbol{Q}_{\mathrm{u}}^{-1} \cdot \overline{\boldsymbol{\sigma}}_{\mathrm{n}}} \times \frac{\dot{e} \boldsymbol{m} \cdot \boldsymbol{\eta}_{\mathrm{n}}}{\boldsymbol{\eta}: \dot{\boldsymbol{\varepsilon}}^{-}+\dot{e} \boldsymbol{m} \cdot \boldsymbol{\eta}_{\mathrm{n}}}
\end{aligned}
$$


The minimum value of the first fraction on the right-hand side of [25] is $g_{c r i t}^{\prime}$, given by [21]. Due to the loading-unloading conditions [23], the minimal value of the second fraction on the right-hand side of [25] is 1, and it is attained if and only if $\boldsymbol{\eta}: \dot{\boldsymbol{\varepsilon}}^{-}=0$. But this characterizes the extreme case in which unloading on the negative side of the discontinuity has the character of neutral loading. So we can conclude that the smallest possible value of $g^{\prime}$ for which a discontinuous bifurcation can occur is $g_{\text {crit }}^{\prime}$, as revealed by analysis of the localization tensor corresponding to the tangent stiffness for growing damage, and that in the most critical case damage grows on one side of the discontinuity while the material on the other side experiences neutral loading. Since neutral loading can be considered at the same time as damage growth (at zero rate) and as elastic unloading, the analysis of cases 2 and 3 has lead to the same conclusion regarding the most critical case. Therefore, the simplified analysis based on the determinant of the localization tensor is fully sufficient.

\subsection{Localization condition for one-dimensional model}

To get more insight into the meaning of the critical value of $g^{\prime}$, let us reduce the results derived so far to the simplest case of a one-dimensional damage model. All tensors become scalars, the elastic stiffness tensor $\boldsymbol{D}_{\mathrm{e}}$ is replaced by Young's modulus $E$, the equivalent strain $\varepsilon_{\text {eq }}$ is the strain $\varepsilon$ itself (we consider monotonic tensile loading), and tensor $\boldsymbol{\eta}$ is replaced by the scalar $\eta=\mathrm{d} \varepsilon_{\text {eq }} / \mathrm{d} \varepsilon=1$. The unit normal vector $\boldsymbol{n}$ is also replaced by the scalar $n=1$, and so there is no difference between the localization tensor and the tangent stiffness. Realizing that the effective stress is $\bar{\sigma}=E \varepsilon$ and substituting into [14] we get the tangent stiffness

$$
E_{\mathrm{ed}}=E_{\mathrm{u}}-g^{\prime} \bar{\sigma} \eta=(1-\omega) E-g^{\prime} E \varepsilon=E\left(1-\omega-g^{\prime} \varepsilon\right)
$$

The localization condition $\operatorname{det} \boldsymbol{Q}_{\text {ed }}=0$ is now written as $E_{\text {ed }}=0$, which means that a discontinuous bifurcation occurs when the peak of the stress-strain curve is reached. This is of course the result that we would expect intuitively, based on the example from section 1 . The value of $g^{\prime}$ corresponding to vanishing tangent stiffness $E_{\text {ed }}$ is

$$
g_{\text {crit }}^{\prime}=\frac{1-\omega}{\varepsilon}
$$

This is exactly what we obtain from the general formula [21] by substituting 1 for $\boldsymbol{\eta}_{\mathrm{n}}$, $(1-\omega) E$ for $\boldsymbol{Q}_{\mathrm{u}}$ and $E \varepsilon$ for $\overline{\boldsymbol{\sigma}}_{\mathrm{n}}$.

\subsection{Localization conditions for three-dimensional models}

Evaluation of the critical value of $g^{\prime}$ was in the one-dimensional case very easy because no maximization with respect to $\boldsymbol{n}$ was needed. Let us now show how to proceed in the three-dimensional case. First, note that all terms in the product $\boldsymbol{\eta}_{\mathrm{n}}$. $\boldsymbol{Q}_{\mathrm{u}}^{-1} \cdot \overline{\boldsymbol{\sigma}}_{\mathrm{n}}$ depend on the unit normal vector $\boldsymbol{n}$. Vectors $\boldsymbol{\eta}_{\mathrm{n}}$ and $\overline{\boldsymbol{\sigma}}_{\mathrm{n}}$ are respectively 
defined as $\boldsymbol{\eta} \cdot \boldsymbol{n}$ and $\overline{\boldsymbol{\sigma}} \cdot \boldsymbol{n}$, where $\boldsymbol{\eta}$ and $\overline{\boldsymbol{\sigma}}$ are, for a given material state, fixed symmetric second-order tensors. The inverse of $\boldsymbol{Q}_{\mathrm{u}}$ is easily evaluated as

$$
\boldsymbol{Q}_{\mathrm{u}}^{-1}=\frac{1}{1-\omega} \boldsymbol{Q}_{\mathrm{e}}^{-1}=\frac{1}{(1-\omega) G}\left(\boldsymbol{\delta}-\frac{\boldsymbol{n} \otimes \boldsymbol{n}}{2(1-\nu)}\right)
$$

where $G \equiv \mu$ is the elastic shear modulus. The function to be maximized with respect to $\boldsymbol{n}$ is thus

$$
f(\boldsymbol{n})=\boldsymbol{\eta}_{\mathrm{n}} \cdot \boldsymbol{Q}_{\mathrm{u}}^{-1} \cdot \overline{\boldsymbol{\sigma}}_{\mathrm{n}}=\frac{1}{(1-\omega) G}\left[\boldsymbol{n} \cdot \boldsymbol{\eta} \cdot \overline{\boldsymbol{\sigma}} \cdot \boldsymbol{n}-\frac{(\boldsymbol{n} \cdot \boldsymbol{\eta} \cdot \boldsymbol{n})(\boldsymbol{n} \cdot \overline{\boldsymbol{\sigma}} \cdot \boldsymbol{n})}{2(1-\nu)}\right]
$$

The principal directions of tensors $\overline{\boldsymbol{\sigma}}=\boldsymbol{D}_{\mathrm{e}}: \boldsymbol{\varepsilon}$ and $\boldsymbol{\eta}=\partial \varepsilon_{\mathrm{eq}} / \partial \boldsymbol{\varepsilon}$ are the same, and if we rewrite [29] in terms of the components with respect to the principal coordinate system, we obtain the fourth-order polynomial

$$
f\left(n_{1}, n_{2}, n_{3}\right)=\frac{1}{(1-\omega) G}\left[\sum_{I=1}^{3} \eta_{I} \bar{\sigma}_{I} n_{I}^{2}-\frac{1}{2(1-\nu)} \sum_{I=1}^{3} \eta_{I} n_{I}^{2} \sum_{I=1}^{3} \bar{\sigma}_{I} n_{I}^{2}\right]
$$

This polynomial has to be maximized, subject to the constraint $n_{1}^{2}+n_{2}^{2}+n_{3}^{2}=1$. Renaming $n_{1}^{2}$ as $N_{1}, n_{2}^{2}$ as $N_{2}$ and $n_{3}^{2}$ as $N_{3}$, we convert the objective function $f$ into a quadratic function of arguments $N_{1}, N_{2}$ and $N_{3}$, and the constraint into a linear one, but additional constraints $N_{1} \geq 0, N_{2} \geq 0$ and $N_{3} \geq 0$ must be imposed. The resulting optimization problem has a strictly concave objective function and a convex admissible domain, and so the solution exists and is unique. It can be obtained by methods of quadratic programming.

In general, the critical value of $g^{\prime}$ depends on the current state, which enters through the principal values of $\boldsymbol{\eta}$ and $\overline{\boldsymbol{\sigma}}$. To illustrate the influence of the particular definition of equivalent strain on the localization properties of the model, we restrict our attention to the case of uniaxial tension, characterized by $\bar{\sigma}_{2}=\bar{\sigma}_{3}=0$ and $\eta_{2}=\eta_{3}$. The objective function simplifies to

$$
f\left(N_{1}, N_{2}, N_{3}\right)=\frac{1}{(1-\omega) G}\left[\eta_{1} \bar{\sigma}_{1} N_{1}-\frac{\left(\eta_{1} N_{1}+\eta_{2}\left(N_{2}+N_{3}\right)\right) \bar{\sigma}_{1} N_{1}}{2(1-\nu)}\right]
$$

and exploiting the constraint $N_{1}+N_{2}+N_{3}=1$ we can express the objective function as function of a single variable,

$$
f\left(N_{1}\right)=\frac{\bar{\sigma}_{1} N_{1}}{(1-\omega) G}\left[\eta_{1}-\frac{\eta_{1} N_{1}+\eta_{2}\left(1-N_{1}\right)}{2(1-\nu)}\right]
$$


This quadratic function of $N_{1}$ has to be maximized on the interval $[0,1]$, to make sure that the resulting components of vector $\boldsymbol{n}$ will be real. A routine procedure leads to the conclusion that

- if $2 \nu \eta_{1} \leq \eta_{2}$, then $f$ attains its maximum value

$$
f_{\max }=\frac{\bar{\sigma}_{1}}{2(1-\omega) G(1-\nu)}(1-2 \nu) \eta_{1}
$$

at $N_{1}=1$,

- if $2 \nu \eta_{1} \geq \eta_{2}$, then $f$ attains its maximum value

$$
f_{\max }=\frac{\bar{\sigma}_{1}}{2(1-\omega) G(1-\nu)} \frac{\left[2(1-\nu) \eta_{1}-\eta_{2}\right]^{2}}{4\left(\eta_{1}-\eta_{2}\right)}
$$

at $N_{1}=\left[2(1-\nu) \eta_{1}-\eta_{2}\right] /\left(2 \eta_{1}-2 \eta_{2}\right)$.

In the first case, the predicted discontinuity plane is perpendicular to the direction of loading, which is what we would expect if a quasibrittle material is cracking under uniaxial tension. In the second case, the discontinuity plane is inclined. The question that naturally arises is what actually happens for typical isotropic damage models. The answer depends on the ratio $\eta_{2} / \eta_{1}$, i.e., on the choice of the formula for equivalent strain.

Mazars (1984) defined the equivalent strain as

$$
\varepsilon_{\mathrm{eq}}=\|\langle\varepsilon\rangle\|=\sqrt{\sum_{I=1}^{3}\left\langle\varepsilon_{I}\right\rangle^{2}}
$$

where $\varepsilon_{I}, I=1,2,3$, are the principal strains, and the brackets $\langle\ldots\rangle$ denote the positive part. Tensor $\boldsymbol{\eta}$ is obtained as

$$
\boldsymbol{\eta}=\frac{\partial \varepsilon_{\mathrm{eq}}}{\partial \varepsilon}=\frac{\langle\boldsymbol{\eta}\rangle}{\varepsilon_{\mathrm{eq}}}
$$

Under uniaxial tension, only one principal strain $\varepsilon_{1}=\bar{\sigma}_{1} / E$ is positive while the other principal strains $\varepsilon_{2}=\varepsilon_{3}=-\nu \varepsilon_{1}$ are negative (provided that the Poisson ratio is positive, which is always the case for usual materials). Therefore, we have $\eta_{1}=1$ and $\eta_{2}=\eta_{3}=0$, condition $2 \nu \eta_{1} \leq \eta_{2}$ is not satisfied and the predicted direction of potential discontinuity plane is inclined (not perpendicular to the loading direction). The angle $\theta$ between the normal to the discontinuity plane and the direction of loading is evaluated from the relation $\cos \theta=n_{1}=\sqrt{N_{1}}$ as

$$
\theta=\arccos \sqrt{1-\nu}
$$

and for instance for $\nu=0.2$ (a typical value for concrete), it is about 27 degrees. This seems to be quite a suprizing result. But even more surprizing is the critical value of 
tangent modulus. For $\eta_{1}=1$ and $\eta_{2}=\eta_{3}=0$, the maximum value of the objective function $f$ is

$$
f_{\max }=\frac{\bar{\sigma}_{1}(1-\nu)}{2(1-\omega) G}=\frac{\left(1-\nu^{2}\right) \varepsilon}{1-\omega}
$$

and if $g_{\text {crit }}^{\prime}=1 / f_{\max }$ is substituted into [26], we obtain

$$
E_{\text {ed,crit }}=E\left(1-\omega-g_{c r i t}^{\prime} \varepsilon\right)=-\frac{\nu^{2} E_{\mathrm{u}}}{1-\nu^{2}}
$$

This means that the discontinuous bifurcation is excluded not only in the hardening regime with positive tangent modulus, but also if the material is softening but the tangent modulus is only "slightly negative". The absolute value of the negative tangent modulus must attain at least $\nu^{2} /\left(1-\nu^{2}\right)$ times the unloading modulus to make the discontinuous bifurcation possible. For Poisson ratio 0.2 , the critical ratio $E_{\mathrm{ed}} / E_{\mathrm{u}}$ is -0.04167 .

Another definition of equivalent strain is based on the scaled energy norm,

$$
\varepsilon_{\mathrm{eq}}=\sqrt{\frac{\varepsilon: D_{e}: \varepsilon}{E}}
$$

In this case we obtain $\boldsymbol{\eta}=\overline{\boldsymbol{\sigma}} / E \varepsilon_{\text {eq }}$ and, under uniaxial tension, $\eta_{1}=1$ and $\eta_{2}=0$. So the localization properties under uniaxial tension are exactly the same as for the Mazars definition.

For the Rankine-type definition of equivalent strain

$$
\varepsilon_{\mathrm{eq}}=\frac{\bar{\sigma}_{1}}{E}
$$

we get

$$
\begin{aligned}
\boldsymbol{\eta} & =\frac{\partial \varepsilon_{\mathrm{eq}}}{\partial \varepsilon}=\frac{1}{E} \frac{\partial \bar{\sigma}_{1}}{\partial \overline{\boldsymbol{\sigma}}}: \frac{\partial \overline{\boldsymbol{\sigma}}}{\partial \boldsymbol{\varepsilon}}=\frac{1}{E}\left(\boldsymbol{p}_{1} \otimes \boldsymbol{p}_{1}\right): \boldsymbol{D}_{e}= \\
& =\frac{1}{1+\nu}\left(\frac{\nu}{1-2 \nu} \boldsymbol{\delta}+\boldsymbol{p}_{1} \otimes \boldsymbol{p}_{1}\right)
\end{aligned}
$$

where $\bar{\sigma}_{1}$ is the maximum principal effective stress and $\boldsymbol{p}_{1}$ is the unit vector in the corresponding principal direction. The ratio $\eta_{2} / \eta_{1}$ is equal to $\nu /(1-\nu)$, which is smaller than $2 \nu$ for all values of Poisson's ratio $\nu$ from the open interval $(0,0.5)$. So once again, the discontinuity plane is not perpendicular to the direction of loading. But in contrast to the previous examples, the critical value of the tangent modulus turns out to be positive-it is equal to $E_{\mathrm{u}} \nu^{2} /(2-\nu)^{2}$. This means that the discontinuous bifurcation can occur even before the peak stress is attained (of course provided that the stress-strain curve has a nonlinear hardening part before the peak, with a gradual decrease of the tangent modulus). For $\nu=0.2$, the critical value of the tangent modulus is about $1 \%$ of the unloading modulus, so it is quite small. Much larger positive 
values are found for a model based on the so-called modified von Mises definition of equivalent strain. This model uses an additional parameter $k$, which sets the ratio between compressive and tensile strength. With $k=10$ and $\nu=0.2$, the critical ratio $E_{\text {ed }} / E_{\mathrm{u}}$ is as high as 0.267 . So if this type of model is used with pre-peak nonlinear behavior, strange instabilities in the hardening regime can be expected.

Localization analysis can also be performed for anisotropic damage models. For instance, for smeared crack models the results in a good agreement with our expectations: The discontinuous bifurcation condition is satisfied at the peak of the tensile stress-strain curve and the discontinuity plane is perpendicular to the direction of loading. This confirms that the model is appropriate for quasibrittle materials such as concrete.

\section{Acknowledgements}

Financial support of the Ministry of Education of the Czech Republic under Research Plan MSM 6840770003 is gratefully acknowledged.

\section{References}

Hadamard J., Leçons sur la propagation des ondes, Librairie Scientifi que A. Hermann et Fils, Paris, 1903.

Hill R., "A general theory of uniqueness and stability in elastic-plastic solids", Journal of the Mechanics and Physics of Solids, vol. 6, 1958, p. 236-249.

Jirásek M., "Nonlocal damage mechanics", Revue Européenne de Génie Civil, vol. 11, 2007, p. 1013-1040.

Mazars J., Application de la mécanique de l'endommagement au comportement non linéaire et à la rupture du béton de structure, Thèse de Doctorat d'Etat, Université Paris VI., France, 1984.

Ottosen N., Runesson K., "Properties of discontinuous bifurcation solutions in elastoplasticity”, International Journal of Solids and Structures, vol. 27, 1991, p. 401-421.

Rizzi E., Carol I., Willam K., "Localization analysis of elastic degradation with application to scalar damage", Journal of Engineering Mechanics, ASCE, vol. 121, 1996, p. 541-554.

Rudnicky J. W., Rice J. R., "Conditions for the localization of deformation in pressure-sensitive dilatant materials", Journal of the Mechanics and Physics of Solids, vol. 23, 1975, p. 371394. 


\section{ANNEXE POUR LE SERVICE FABRICATION \\ A FOURNIR PAR LES AUTEURS AVEC UN EXEMPLAIRE PAPIER \\ DE LEUR ARTICLE ET LE COPYRIGHT SIGNE PAR COURRIER \\ LE FICHIER PDF CORRESPONDANT SERA ENVOYE PAR E-MAIL}

1. ARTICLE POUR LA REVUE :

REGC - 11/2007. Damage and fracture in geomaterials

2. Auteurs :

Milan Jirásek

3. TITRE DE L'ARTICLE :

Mathematical analysis of strain localization

4. Titre ABRÉGÉ POUR LE HAUT DE PAGE MOINS DE 40 Signes :

Analysis of strain localization

5. DATE DE CETTE VERSiON :

September 17, 2007

6. CoOrdonnéEs des Auteurs :

- adresse postale :

Department of Mechanics

Faculty of Civil Engineering

Czech Technical University in Prague

16629 Prague, Czech Republic

Milan.Jirasek@epfl.ch

- téléphone : +420-224354481

- télécopie : +420-224310775

- e-mail : milan.jirasek@fsv.cvut.cz

7. LOGICIEL UTILISÉ POUR LA PRÉPARATION DE CET ARTICLE :

IATEX, avec le fichier de style article-hermes2.cls, version $1.23 \mathrm{du}$ 02/08/2006.

8. FORMULAIRE DE COPYRIGHT :

Retourner le formulaire de copyright signé par les auteurs, téléchargé sur : http://www. revuesonline.com

SERVICE ÉDITORIAL - HERMES-LAVOISIER 14 rue de Provigny, F-94236 Cachan cedex

Tél. : 01-47-40-67-67

E-mail : revues@lavoisier.fr

Serveur web : http://www.revuesonline.com 